HIP-2001-61/TH

\title{
BRST properties of New Superstring States
}

\author{
Dimitri Polyakov ${ }^{\dagger}$ \\ Dept. of Physical Sciences \\ Helsinki University \\ and Helsinki Institute of Physics \\ $P L 64$ \\ FIN-00014 Helsinki, Finland
}

\begin{abstract}
Brane-like states are defined by physical vertex operators in NSR superstring theory, existing at nonzero pictures only. These states exist both in open and closed string theories, in the NS and NS-NS sectors respectively. In this paper we present a detailed analysis of their BRST properties, giving a proof that these vertex operators are physical, i.e. BRST invariant and BRST non-trivial. Keywords: PACS:04.50. $+h ; 11.25 . M j$.
\end{abstract}

November 2001

$\dagger$ polyakov@mappi.helsinki.fi 


\section{Introduction}

Critical superstring theory in the NSR formalism contains physical vertex operators refs[1], [2] with ghost-matter mixing, i.e. the operators that exist at non-zero pictures only. These vertex operators do not correspond to any point-like particle in the perturbative superstring spectrum but instead are closely related to the dynamics of extended objects, such as D-branes. These operators are therefore expected to play a crucial role in nonperturbative physics of strings and somehow it appears that it is an essential mixing of matter with superconformal ghosts in these vertex operators that is relevant to their nonperturbative nature. In the open string case, these massless vertex operators, one two-form (at picture -2 ) and two five-forms (at pictures -3 and +1 ), are given by:

$$
\begin{array}{r}
V_{5}^{(-3)}=\oint \frac{d z}{2 i \pi} e^{-3 \phi} \psi_{m_{1}} \ldots \psi_{m_{5}} e^{i k^{\perp} X}(z) \\
V_{5}^{(+1)}=\oint \frac{d z}{2 i \pi} e^{\phi} \psi_{m_{1}} \ldots \psi_{m_{5}} e^{i k^{\perp} X}+\text { ghosts } \\
V_{2}^{(-2)}=\oint \frac{d z}{2 i \pi} e^{-2 \phi} \psi_{m_{1}} \psi_{m_{2}} e^{i k^{\perp} X}
\end{array}
$$

Bosonization expressions for superconformal ghosts are the standard ones; see the formula (88) of this paper. The contour integrals in (1) are taken over the open string worldsheet boundary; $\psi$ 's are NSR worldsheet fermions and $\phi$ is bosonized superconformal ghost field; the space-time indices $m_{i}$ run from 0 to 9 . The picture +1 five-form vertex also contains fermionic ghost part (consisting of two terms with fermionic ghost numbers +1 and -1 ); these fermionic ghost terms are necessary to insure the BRST invariance of this vertex operator; in this paper we will particularly give a detailed derivation of this fermionic ghost part. One important property of the brane-like states distinguishing them from usual vertex operators in ten-dimensional string theory, such as a photon or a graviton, is their propagation: namely, as we will show in details in Section 2 of this paper, the BRST invariance and non-triviality conditions altogether restrict their propagation to lower dimensional subspaces; that is, their momenta $k^{\perp}$ must be taken transverse with respect to their $m_{i}$ space-time indices. Thus $V_{5}^{(+1)}$ and $V_{5}^{(-3)}$ are constrained to propagate in fivedimensional subspace of $R^{10}$ while the two-form is allowed to propagate in eight dimensions. In this respect, the brane-like states (1) are reminiscent of a multi-dimensional analogue of the discrete states in two-dimensional gravity [3]. The closed string brane-like states may be constructed from the open string ones by multiplying them by a suitable antiholomorphic part; there are in fact various ways of doing that corresponding to various 
brane configurations. As usual, the vertex operators for the open-string brane-like states may also be written in an alternative, unintegrated form:

$$
\begin{array}{r}
V_{5}^{(-3)}=c e^{-3 \phi} \psi_{m_{1}} \ldots \psi_{m_{5}} e^{i k^{\perp} X}(z) \\
V_{5}^{(+1)}=c e^{\phi} \psi_{m_{1}} \ldots \psi_{m_{5}} e^{i k^{\perp} X}+\text { ghosts } \\
V_{2}^{(-2)}=c e^{-2 \phi} \psi_{m_{1}} \psi_{m_{2}} e^{i k^{\perp} X}
\end{array}
$$

where $c$ is fermionic ghost field.

In this paper we perform the detailed analysis of the BRST properties of the branelike vertex operators proving that they correspond to well-defined physical states in the NSR This paper is organized as follows. In the first section we review arguments relating the brane-like states to D-brane dynamics. We show that zero momentum parts of these operators appear as central terms in picture-changed superalgebras, thus corresponding to brane topological charges. We also demonstrate that these vertex may be considered as a special limit of the RR states in non-canonical pictures. In the sections 2 and 3 we analyze BRST properties of the brane-like states. In the section 2 we prove the BRST invariance of the brane-like states $(1),(2)$. While for the two-form and the 5 -form at the picture -3 the proof is elemenrtary and straightforward, to insure the BRST invariance of the picture +1 five-form one has to introduce two fermionic ghost counterterms, carrying fermionic ghost numbers +1 and -1 . We give the detailed derivation of these counterterms and show that their ghost structure is similar to the one present in the BRST invariant expression for picture-changing operator 4 Next, in the section 3 we give the proof that the states (1), (2), are BRST-nontrivial, i.e. cannot be represented as a BRST commutator with any operator in a small Hilbert space. At the same time, the BRST non-triviality condition will be shown to impose significant constraints on propagation of the 5-form state. Unlike the case of conventional perturbative vertex operators in critical superstring theory propagating in 10 dimensions, such as a graviton or a photon, the five-form state will be shown to be constrained to propagate in the five-dimensional subspace, transverse to the one spanned by the $m_{1}, \ldots m_{5}$ indices. Thus in this paper we give complete and detailed proof that the new superstring states (1) are physical and therefore must play an important role in non-perturbative superstring dynamics. In the concluding section we construct closed-string version of the brane-like state by a straightforward generalization of an open string case, and also present some new results on the contribution of the brane-like states (1) to the low-energy effective action of superstring theory to demonstrate that it reproduces the DBI expression for D-brane actions. 


\section{Brane-like States and Central Terms in space-time SUSY algebras}

In this section we will show that the vertices (1) at zero momentum appear as central terms in picture-changed superalgebras in the presence of D-branes, which points at their relation to brane dynamics. Consider the space-time supercharge at the canonical $-1 / 2$ picture:

$$
Q_{\alpha}^{-1 / 2}=\oint \frac{d z}{2 i \pi} e^{-\frac{\phi}{2}} \Sigma_{\alpha}(z)
$$

where $\alpha, \beta, \ldots$ denote $10 \mathrm{~d}$ spinor indices. It is easy to check that this supercharge satisfies the standard SUSY algebra:

$$
\left\{Q_{\alpha}^{-1 / 2} ; Q_{\beta}^{-1 / 2}\right\}=\gamma_{\alpha \beta}^{m} P_{m}^{(-1)}
$$

where

$$
P_{m}^{(-1)}=\oint \frac{d z}{2 i \pi} e^{-\phi} \psi_{m}
$$

is a translation operator at the -1-picture. Consider, however, a deformation of this supercharge by a BRST exact space-time spinor, $T_{\alpha}=e^{-\frac{3 \phi}{2}} \Sigma_{\alpha}(z)$ Note that even though $T_{\alpha}$ is BRST exact, its propagation is described by a physical state $T_{\alpha} e^{i k X}$ which is BRST nontrivial at nonzero momentum. In particular, it is well-known that the Ramond-Ramond physical state, $T \bar{S} e^{i k X}$ is the source of the RR gauge potential $A_{R R}(k)$, rather than the RR field strength (unlike the canonical picture case). Denoting the deformed space-time SUSY charge as $S_{\alpha}=Q_{\alpha}+T_{\alpha}$, one can easily show that it satisfies the superalgebra:

$$
\left\{S_{\alpha} ; S_{\beta}\right\}=\gamma_{\alpha \beta}^{m} P_{m}^{(-1)}+\gamma_{\alpha \beta}^{m_{1} m_{2}} Z_{m_{1} m_{2}}+\gamma_{\alpha \beta}^{m_{1} \ldots m_{5}} Z_{m_{1} \ldots m_{5}}
$$

where the 2-form and the 5-form central terms are given by

$$
\begin{array}{r}
Z_{m_{1} m_{2}}=\oint \frac{d z}{2 i \pi} e^{-2 \phi} \psi_{m_{1}} \psi_{m_{2}} \\
Z_{m_{1} \ldots m_{5}}=\oint \frac{d z}{2 i \pi} e^{-3 \phi} \psi_{m_{1}} \ldots \psi_{m_{5}}
\end{array}
$$

i.e. they are given by the zero momentum parts of the vertices (1). It is well-known that p-form central terms in SUSY algebra always correspond to topological charges of p-branes, i.e. non-perturbative extended objects. Therefore we expect that the vertex operators (1), if they are physical, must describe the non-perturbative brane dynamics. The non-perturbative nature of these operators should be related to the fact that they don't exist at zero picture, i.e. to the ghost-matter mixing. 
Because of the topological nature of the central charges (7), and because of the BRST triviality of $T_{\alpha}$ one should expect that the operators (1) are BRST exact at zero momentum, however they should be physical at nonzero $k$, just like in the case of picture $-3 / 2$ spacetime spinor $T_{\alpha} e^{i k X}$. In the sections 2 and 3 of the paper we will prove that this is precisely the case, i.e. the operators (1) at $k \neq 0$ indeed define physical superstring excitations. Finally, the vertices (1) may be understood as a special singular limit of Ramond-Ramond states at non-canonical pictures. Indeed, consider the $\mathrm{RR}$ vertices:

$$
\begin{aligned}
& V_{R R}^{-3 / 2,-3 / 2}=T_{\alpha} \bar{T}_{\beta} e^{i k X}(z, \bar{z}) \\
& V_{R R}^{-3 / 2,-1 / 2}=T_{\alpha} \bar{S}_{\beta} e^{i k X}(z, \bar{z})
\end{aligned}
$$

(of course they should be multiplied by appropriate combinations of $10 \mathrm{~d}$ gamma-matrices and Ramond-Ramond tensor fields). Consider these operators placed on the disc, i.e. in some D-brane background. Because of the disc boundaries, the holomorphic and antiholomorphic parts of these operators are no longer independent but have a non-trivial O.P.E. product. Whenever any of these operators approaches the edge of the D-brane, or the disc boundary, this O.P.E. becomes singular, proportional to $\frac{1}{z-\bar{z}}$ multiplied by some boundary operator. It is easy to check (as the calculation is totally similar to computing the anticommutators in the SUSY algebra (6)) that this boundary operator is given exactly by the two-form $Z_{m_{1} n_{2}} e^{i k X}$ in case of the $V_{R R}^{-3 / 2,-1 / 2} \mathrm{RR}$ operator, and by the five-form $Z_{m_{1} \ldots m_{5}} e^{i k X}$ in case of $V_{R R}^{-3 / 2,-3 / 2}$, multiplied by suitable products of $10 \mathrm{~d}$ gamma-matrices. Thus the appearance of the operators (1) can be understood as a result of a normal reordering inside the Ramond-Ramond vertices taking place when the RR charge sources are located near the D-brane edge. In particular, this is an example of an interesting connection between D-brane Ramond-Ramond and topological charges.

\section{BRST-invariance of the Brane-like States}

In this section, we demonstrate the BRST invariance of the brane-like vertex operators as a first necessary step to show that they define physical string-theoretic states. The BRST operator is given by:

$$
Q_{B R S T}=\oint \frac{d z}{2 i \pi}\left(c\left(T_{\text {matter }}+T_{\text {ghost }}\right)+\frac{1}{2} e^{\phi-\chi} \psi_{m} \partial X^{m}+\frac{1}{4} e^{2 \phi-2 \chi} b-b: c \partial c:\right)
$$

Let us first of all analyze the question of the BRST invariance of the vertices (1),(2). Let us consider the integrated vertices (1). It is easy to see that the $V_{5}^{(-3)}$ and $V_{2}^{(-2)}$ 
operators, i.e. the picture -3 five-form and the two-form are BRST-invariant. indeed, since the integrands of $V_{5}^{(-3)}$ and $V_{2}^{(-2)}$ are primary fields of conformal dimension 1 , we have

$$
\begin{aligned}
& \left\{\oint \frac{d z}{2 i \pi} c\left(T_{\text {matter }}+T_{\text {ghost }}(z), V_{5}^{(-3)}\right\}\right. \\
& =\oint \frac{d w}{2 i \pi}\left[\oint \frac{d z}{2 i \pi} \frac{1}{(z-w)^{2}}: c e^{-3 \phi} \psi_{m_{1}} \ldots \psi_{m_{5}} e^{i k^{\perp} X}:(w)\right. \\
& +\frac{1}{(z-w)}\left(: \partial c e^{-3 \phi} \psi_{m_{1}} \ldots \psi_{m_{5}} e^{i k^{\perp} X}:(w)+: c \partial\left(e^{-3 \phi} \psi_{m_{1}} \ldots \psi_{m_{5}} e^{i k^{\perp} X}:(w)\right)\right] \\
& =\oint \frac{d w}{2 i \pi}(\partial c) e^{-3 \phi} \psi_{m_{1}} \ldots \psi_{m_{5}} e^{i k^{\perp} X}+c \partial\left(e^{-3 \phi} \psi_{m_{1}} \ldots \psi_{m_{5}} e^{i k^{\perp} X}\right) \\
& =\oint \frac{d w}{2 i \pi} \partial\left(c e^{-3 \phi} \psi_{m_{1}} \ldots \psi_{m_{5}} e^{i k^{\perp} X}\right)=0
\end{aligned}
$$

Next, using the O.P.E's:

$$
\begin{aligned}
: e^{\alpha \phi}:(z): e^{\beta \phi}:(w) & \sim(z-w)^{\alpha \beta}: e^{(\alpha+\beta) \phi}:(w) \\
: e^{\alpha \chi}:(z): e^{\beta \chi}:(w) \sim(z-w)^{-\alpha \beta}: e^{(\alpha+\beta) \chi}:(w) b(z) c(w) & \sim \frac{1}{z-w}+\ldots \\
c(z) c(w)) & \sim(z-w): c \partial c:(w)+\ldots \\
b(z) b(w)) & \sim(z-w): b \partial b:(w)+\ldots
\end{aligned}
$$

it is easy to see that other terms in the BRST current have no poles in the O.P.E. with $e^{-3 \phi} \psi_{m_{1}} \ldots \psi_{m_{5}} e^{i k^{\perp} X}(w)$ and therefore

$$
\left\{\oint \frac{d z}{2 i \pi}\left(\frac{1}{2} e^{\phi-\chi} \psi_{m} \partial X^{m}+\frac{1}{4} e^{2 \phi-2 \chi} b-b: c \partial c:\right), V_{5}^{(-3)}\right\}=0
$$

and hence

$$
\left\{Q_{B R S T}, V_{5}^{(-3)}\right\}=0
$$

The BRST invariance of the two-form $V_{5}^{(-3)}$ is proven totally analogously. However, for the picture +1 five-form things are more subtle. The straightforward generalization, simply by replacing the $e^{-3 \phi}$ of $V_{5}^{(-3)}$ with the $e^{\phi}$ field having the superconformal ghost number +1 and of the same conformal dimension $-\frac{3}{2}$, is not BRST invariant, since the $e^{-3 \phi} \psi_{m_{1}} \ldots \psi_{m_{5}} e^{i k^{\perp} X}:(w)$ operator does not commute with the supercurrent part of the BRST charge given by the terms $\frac{1}{2} e^{\phi-\chi} \psi_{m} \partial X^{m}$ and $\frac{1}{4} e^{2 \phi-2 \chi} b$ of $Q_{B R S T}$. Namely, calculating the terms giving the simple poles in the relevant O.P.E's and evaluating the residue, one easily gets

$$
\begin{aligned}
& \left\{\oint \frac{d z}{2 i \pi} e^{\phi-\chi} \psi_{m} \partial X^{m}(z), e^{\phi} \psi_{m_{1}} \ldots \psi_{m_{5}} e^{i k^{\perp} X}:(w)\right\} \\
& =e^{2 \phi-\chi}\left\{\psi_{m_{1}} \ldots \psi_{m_{5}}(\psi \partial X)^{\perp}+\psi_{\left[m_{1} \ldots \psi_{m_{4}} \partial X_{m_{5}}\right]}(\partial \phi-\partial \chi)\right. \\
& \left.+\psi_{\left[m_{1}\right.} \ldots \psi_{m_{4}} \partial^{2} X_{\left.m_{5}\right]}+i \psi_{m_{1}} \ldots \psi_{m_{5}}\left(\left(k^{\perp} \psi\right)(\partial \phi-\partial \chi)+\left(k^{\perp} \partial \psi\right)\right)\right\} e^{i k^{\perp} X}:(w)
\end{aligned}
$$


and

$$
\begin{array}{r}
\oint \frac{d z}{2 i \pi}\left\{e^{2 \phi-2 \chi} b(z), e^{\phi} \psi_{m_{1}} \ldots \psi_{m_{5}} e^{i k^{\perp} X}(w)\right\} \\
=b e^{3 \phi-2 \chi} \psi_{m_{1}} \ldots \psi_{m_{5}}(2 \partial \phi+2 \partial \chi-\partial \sigma) e^{i k^{\perp} X}(w)
\end{array}
$$

therefore

$$
\begin{aligned}
& \left\{Q_{B R S T}, e^{\phi} \psi_{m_{1}} \ldots \psi_{m_{5}} e^{i k^{\perp} X}:(w)\right\} \\
& =\frac{1}{2} e^{2 \phi-\chi}\left\{\psi_{m_{1}} \ldots \psi_{m_{5}}(\psi \partial X)^{\perp}+\psi_{\left[m_{1}\right.} \ldots \psi_{m_{4}} \partial X_{\left.m_{5}\right]}(\partial \phi-\partial \chi)\right. \\
& \left.+\psi_{\left[m_{1}\right.} \ldots \psi_{m_{4}} \partial^{2} X_{\left.m_{5}\right]}+i \psi_{m_{1}} \ldots \psi_{m_{5}}\left(\left(k^{\perp} \psi\right)(\partial \phi-\partial \chi)+\left(k^{\perp} \partial \psi\right)\right)\right\} e^{i k^{\perp} X}:(w) \\
& +\frac{1}{4} b e^{3 \phi-2 \chi} \psi_{m_{1}} \ldots \psi_{m_{5}}(2 \partial \phi+2 \partial \chi-\partial \sigma) e^{i k^{\perp} X}(w)
\end{aligned}
$$

Here and anywhere else $\left[m_{1} \ldots m_{5}\right]$ denotes antisymmetrization over the the $m_{1}, \ldots, m_{5}$ space-time indices. Therefore the naive expression for the picture +1 five-form, given by $e^{\phi} \psi_{m_{1}} \ldots \psi_{m_{5}} e^{i k^{\perp} X}(w)$ is not BRST-invariant. However, below we will show that BRST invariance is restores if one adds two counterterms to this operators, carrying fermionic ghost numbers +1 and -1 , so that the full expression for the BRST-invariant $V_{5}^{(+1)}$ has the ghost structure similar to the expression for the picture-changing operator. Our strategy to find the appropriate counterterms is the following. Writing schematically

$$
\begin{array}{r}
{\left[\oint \frac{d z}{2 i \pi} e^{\phi-\chi} \psi_{m} \partial X^{m}(z), e^{\phi} \psi_{m_{1}} \ldots \psi_{m_{5}} e^{i k^{\perp} X}:(w)\right]=A_{1}(w)} \\
{\left[\oint \frac{d z}{2 i \pi} e^{2 \phi-2 \chi} b(z), e^{\phi} \psi_{m_{1}} \ldots \psi_{m_{5}} e^{i k^{\perp} X}(w)\right]=A_{2}(w)}
\end{array}
$$

we shall aim to find the counterterms $C_{1}$ and $C_{2}$ such that

$$
\begin{aligned}
{\left[\oint \frac{d z}{2 i \pi} e^{\phi-\chi} \psi_{m} \partial X^{m}(z), C_{1}(w)\right] } & =-\frac{1}{2} A_{2}(w) \\
{\left[\oint \frac{d z}{2 i \pi} e^{2 \phi-2 \chi} b(z), C_{2}(w)\right] } & =-2 A_{1}(w)
\end{aligned}
$$

and such that at the same time both $C_{1}$ and $C_{2}$ commute with all other terms of $Q_{B R S T}$ (such as the energy momentum terms and the "opposite" respective supercurrent term). Then it is easy to see that the combination $V_{5}^{(+1)}=e^{\phi} \psi_{m_{1}} \ldots \psi_{m_{5}} e^{i k^{\perp} X}(w)+C_{1}(w)+C_{2}(w)$ will be BRST invariant. It is also clear that the $C_{1}$ operator must contain the ghost factor of the type $\sim b e^{2 \phi-\chi}(w)$ while the $C_{2}$-operator must be proportional to the factor of the type $\sim c e^{\chi}(w)$, in other words the BRST invariant vertex operator $V_{5}^{(+1)}$ should have the 
ghost structure analogous to that of the picture-changing operator. Let us start with the derivation of $C_{2}$. The ansatz we propose is given by:

$$
\begin{aligned}
& C_{2}(w)=-2 \hat{b_{3}} c \partial c e^{\chi} \partial \chi\left(\psi_{m_{1}} \ldots \psi_{m_{5}}(\psi \partial X)^{\perp}+\psi_{\left[m_{1}\right.} \ldots \psi_{m_{4}} \partial X_{\left.m_{5}\right]}(\partial \phi-\partial \chi)\right. \\
& \left.+\psi_{\left[m_{1}\right.} \ldots \psi_{m_{4}} \partial^{2} X_{\left.m_{5}\right]}+i \psi_{m_{1}} \ldots \psi_{m_{5}}\left(\left(k^{\perp} \psi\right)(\partial \phi-\partial \chi)+\left(k^{\perp} \partial \psi\right)\right)\right) e^{i k^{\perp} X}(w)
\end{aligned}
$$

and the action of the $\hat{b}_{n}$-operator on any operator $A$ located at a point $w$ is defined as

$$
\hat{b}_{n} A(w)=\lim _{u \rightarrow w}\left[\oint \frac{d z}{2 i \pi}(z-u)^{n+1} b(z), A(w)\right]
$$

If the $A$-operator is located at zero point $w=0$, then $\hat{b}_{n}=b_{n}$ is just the nth oscillation mode of the $\mathrm{b}$ ghost field; while at an arbitrary point $w \hat{b}_{n}$ is related to $b_{n}$ by appropriate conformal transformation. Let us now evaluate the commutator of $Q_{B R S T}$ with $C_{2}$. Let us start with the commutator of $C_{2}$ with the ghost supercurrent term of $Q_{B R S T}$ given by $\frac{1}{4} \oint \frac{d z}{2 i \pi} e^{2 \phi-2 \chi} b(z)$. First of all, since there are no singularities in the O.P.E of b with itself, it is easy to see that $\left\{\oint \frac{d z}{2 i \pi} e^{2 \phi-2 \chi} b(z), \hat{b}_{3}\right\}=0$ and therefore

$$
\begin{aligned}
& {\left[\oint \frac{d z}{2 i \pi} e^{2 \phi-2 \chi} b(z), C_{2}(w)\right]} \\
& =-2 \hat{b_{3}}\left\{\oint \frac{d z}{2 i \pi} e^{2 \phi-2 \chi} b(z), c \partial c e^{\chi} \partial \chi\left(\psi_{m_{1}} \ldots \psi_{m_{5}}(\psi \partial X)^{\perp}+\psi_{\left[m_{1}\right.} \ldots \psi_{m_{4}} \partial X_{\left.m_{5}\right]}(\partial \phi-\partial \chi)\right.\right. \\
& \left.+\psi_{\left[m_{1}\right.} \ldots \psi_{m_{4}} \partial^{2} X_{\left.m_{5}\right]}+i \psi_{m_{1}} \ldots \psi_{m_{5}}\left(\left(k^{\perp} \psi\right)(\partial \phi-\partial \chi)+\left(k^{\perp} \partial \psi\right)\right)\right) e^{i k^{\perp} X}(w)
\end{aligned}
$$

Then,

$$
\begin{aligned}
& L_{1} \equiv \\
& \equiv\left\{\oint \frac{d z}{2 i \pi} e^{2 \phi-2 \chi} b(z) ; c \partial c e^{\chi} \partial \chi\left(\psi_{m_{1}} \ldots \psi_{m_{5}}(\psi \partial X)^{\perp}+\psi_{\left[m_{1}\right.} \ldots \psi_{m_{4}} \partial X_{\left.m_{5}\right]}(\partial \phi-\partial \chi)\right.\right. \\
& \left.+\psi_{\left[m_{1}\right.} \ldots \psi_{m_{4}} \partial^{2} X_{\left.m_{5}\right]}+i \psi_{m_{1}} \ldots \psi_{m_{5}}\left(\left(k^{\perp} \psi\right)(\partial \phi-\partial \chi)+\left(k^{\perp} \partial \psi\right)\right)\right) e^{i k^{\perp} X}(w) \\
& =\left(2 P_{2 \phi-2 \chi-\sigma}^{4} c+P_{2 \phi-2 \chi-\sigma}^{3}(2 \partial c+c \partial \chi)\right) e^{2 \phi-\chi} \\
& \times\left(\psi_{m_{1}} \ldots \psi_{m_{5}}(\psi \partial X)^{\perp}+\psi_{\left[m_{1}\right.} \ldots \psi_{m_{4}} \partial X_{\left.m_{5}\right]}(\partial \phi-\partial \chi)\right. \\
& \left.\left.+\psi_{\left[m_{1}\right.} \ldots \psi_{m_{4}} \partial^{2} X_{\left.m_{5}\right]}+i \psi_{m_{1}} \ldots \psi_{m_{5}}\left(\left(k^{\perp} \psi\right)(\partial \phi-\partial \chi)+\left(k^{\perp} \partial \psi\right)\right)\right) e^{i k^{\perp} X}(w)\right\}
\end{aligned}
$$

where the polynomials $P_{\alpha \phi+\beta \chi+\gamma \sigma}^{n}$ are defined as:

$$
e^{\alpha \phi+\beta \chi+\gamma \sigma}(z)=e^{\alpha \phi+\beta \chi+\gamma \sigma}(w)\left(1+\sum_{n=1}^{\infty}(z-w)^{n} P_{\alpha \phi+\beta \chi+\gamma \sigma}^{n}(w)\right)
$$


for any numbers $\alpha, \beta, \gamma$ and in particular

$$
\begin{array}{r}
P_{2 \phi-2 \chi-\sigma}^{4}=\frac{1}{24}\left\{(2 \partial \phi-2 \partial \chi-\partial \sigma)^{4}+(2 \partial \phi-2 \partial \chi-\partial \sigma)^{\prime \prime \prime}\right. \\
+4(2 \partial \phi-2 \partial \chi-\partial \sigma)(2 \partial \phi-2 \partial \chi-\partial \sigma)^{\prime \prime}+3\left((2 \partial \phi-2 \partial \chi-\partial \sigma)^{\prime}\right)^{2} \\
\left.+6(2 \partial \phi-2 \partial \chi-\partial \sigma)^{2}(2 \partial \phi-2 \partial \chi-\partial \sigma)^{\prime}\right\} \\
P_{2 \phi-2 \chi-\sigma}^{3}=\frac{1}{6}\left\{3(2 \partial \phi-2 \partial \chi-\partial \sigma)(2 \partial \phi-2 \partial \chi-\partial \sigma)^{\prime}\right. \\
\left.+(2 \partial \phi-2 \partial \chi-\partial \sigma)^{3}+(2 \partial \phi-2 \partial \chi-\partial \sigma)^{\prime \prime}\right\}
\end{array}
$$

Finally, to compute the commutator of $\frac{1}{4} \oint \frac{d z}{2 i \pi} e^{2 \phi-2 \chi} b(z)$ with $C_{2}$ we need to act on $L_{1}$ with $\hat{b_{3}}$. Using the O.P.E's:

$$
\begin{aligned}
b(z) c(w) & \sim \frac{1}{z-w}+\ldots \\
b(z) \partial \sigma(w) & \sim \frac{b(w)}{z-w}+\ldots \\
b(z) \partial^{2} \sigma(w) & \sim \frac{b(w)}{(z-w)^{2}}+\ldots \\
b(z)(\partial \sigma(w))^{2} & \sim \frac{b(w)}{(z-w)^{2}}+\ldots \\
b(z) \partial^{4} \sigma(w) & \sim \frac{6 b(w)}{(z-w)^{4}}+\ldots \\
b(z)(\partial \sigma(w))^{4} & \sim \frac{b(w)}{(z-w)^{4}}+\ldots
\end{aligned}
$$

we obtain:

$$
\begin{aligned}
& \hat{b}_{3} L_{1}(w)=\frac{1}{2} e^{2 \phi-\chi}\left(\psi_{m_{1}} \ldots \psi_{m_{5}}(\psi \partial X)^{\perp}+\psi_{\left[m_{1}\right.} \ldots \psi_{m_{4}} \partial X_{\left.m_{5}\right]}(\partial \phi-\partial \chi)\right.
\end{aligned}
$$

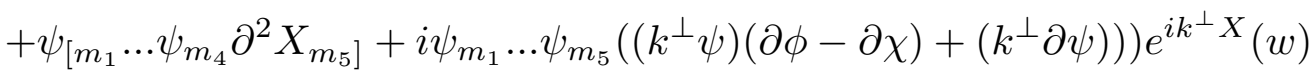

$$
\begin{aligned}
& \equiv \frac{1}{2}\left[\oint \frac{d z}{2 i \pi} e^{\phi-\chi} \psi_{m} \partial X^{m}(z) ; e^{\phi} \psi_{m_{1}} \ldots \psi_{m_{5}} e^{i k^{\perp} X}:(w)\right]
\end{aligned}
$$

Now we will show that $C_{2}$ does commute with all other terms of $Q_{B R S T}$. Indeed, let us consider first the commutator of $C_{2}$ with $\oint \frac{d z}{2 i \pi} e^{\phi-\chi} \psi_{m} \partial X^{m}(z)$. Again, as before, since

$$
\left\{\oint \frac{d z}{2 i \pi} e^{\phi-\chi} \psi_{m} \partial X^{m}(z), \hat{b}_{3}\right\}=0
$$


we write

$$
\begin{aligned}
& {\left[\oint \frac{d z}{2 i \pi} e^{\phi-\chi} \psi_{m} \partial X^{m}(z), C_{2}(w)\right]} \\
& =-4 \hat{b}_{3}\left\{\oint \frac{d z}{2 i \pi} e^{\phi-\chi} \psi_{m} \partial X^{m}(z), c \partial c e^{\chi} \partial \chi\left(\psi_{m_{1}} \ldots \psi_{m_{5}}(\psi \partial X)^{\perp}\right.\right.
\end{aligned}
$$

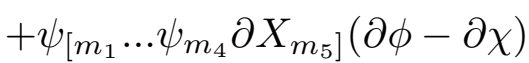

$$
\begin{aligned}
& \left.\left.+\psi_{\left[m_{1}\right.} \ldots \psi_{m_{4}} \partial^{2} X_{\left.m_{5}\right]}+i \psi_{m_{1}} \ldots \psi_{m_{5}}\left(\left(k^{\perp} \psi\right)(\partial \phi-\partial \chi)+\left(k^{\perp} \partial \psi\right)\right)\right) e^{i k^{\perp} X}(w)\right\} \\
& =-4: \hat{b}_{3} \gamma c \partial c F_{\text {matter }}(X, \psi) F_{\text {ghost }}(\phi, \chi):(w) \\
& =-4:\left(\hat{b}_{3} c \partial c\right):(w): \gamma F_{1}(X, \psi, \phi, \chi):(w)
\end{aligned}
$$

where $F_{1}(X, \psi, \phi, \chi)$ denotes the expression that depends entirely on matter fields $X, \psi$ and bosonized superconformal ghosts $\phi, \chi$, i.e. it contains no dependence on the fermionic ghost fields. The precise expression for $F_{1}(X, \psi, \phi, \chi)$ is skipped for the sake of shortness since it is acted on trivially by $\hat{b}_{3}$ and plays no role in further calculations. Now, we have

$$
\begin{array}{r}
: \hat{b}_{3} c \partial c:(w)=\lim _{u \rightarrow w}\left[\oint \frac{d z}{2 i \pi}(z-u)^{4} b(z),: c \partial c:(w)\right] \\
=\lim _{u \rightarrow w}\left(-4 c(w)(u-w)^{3}+\partial c(w)(u-w)^{4}\right)=0
\end{array}
$$

and therefore

$$
\left[\oint \frac{d z}{2 i \pi} e^{\phi-\chi} \psi_{m} \partial X^{m}(z), C_{2}(w)\right]=0
$$

Finally, let us show that the commutator of $C_{2}$ with the stress-energy part of $Q_{B R S T}=$ $\oint \frac{d w}{2 i \pi}(c T-b: c \partial c:)(w)$ also vanishes. Again, as previously we have

$$
\left\{\hat{b}_{3} \oint \frac{d w}{2 i \pi}(c T-b: c \partial c:)(w)\right\}=\lim _{u \rightarrow w} \oint \frac{d w}{2 i \pi}\left(-12(u-w)^{2}+O\left((u-w)^{3}\right)\right)=0
$$

and therefore, using the fact that $\left[\oint \frac{d z}{2 i \pi}(c T-b: c \partial c:)(z), c \partial c(w)\right]=0$ we write

$$
\begin{aligned}
& {\left[\oint \frac{d z}{2 i \pi}(c T-b: c \partial c:)(z), C_{2}(w)\right]} \\
& =-4 \hat{b}_{3}\left\{\oint \frac{d z}{2 i \pi}(c T-b: c \partial c:)(z) ; c \partial c e^{\chi} \partial \chi\left(\psi_{m_{1}} \ldots \psi_{m_{5}}(\psi \partial X)^{\perp}\right.\right.
\end{aligned}
$$

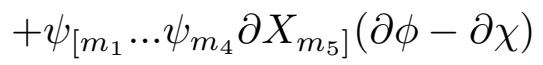

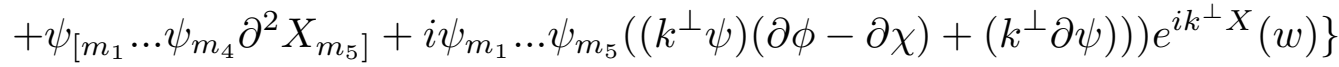

$$
\begin{aligned}
& =-4 \hat{b}_{3} c \partial c \partial^{2} c F(X, \psi, \phi, \chi)(w)=-4:\left(\hat{b}_{3} c \partial c \partial^{2} c\right):(w): F(X, \psi, \phi, \chi):(w) \\
& =-4: F(X, \psi, \phi, \chi):(w) \times \lim _{u \rightarrow w}\left(12(u-w)^{2} c \partial c\right. \\
& \left.-4(u-w)^{3} c \partial^{2} c+(u-w)^{4} \partial c \partial^{2} c\right)=0
\end{aligned}
$$


and thus

$$
\left[\oint \frac{d z}{2 i \pi}(c T-b: c \partial c:)(z), C_{2}(w)\right]=0
$$

Finally, putting together the pieces $(19),(28),(30)$ and (33) we have

$$
\begin{aligned}
& {\left[Q_{B R S T}, C_{2}(w)\right]=-\frac{1}{2} e^{2 \phi-\chi}\left(\psi_{m_{1}} \ldots \psi_{m_{5}}(\psi \partial X)^{\perp}+\psi_{\left[m_{1}\right.} \ldots \psi_{m_{4}} \partial X_{\left.m_{5}\right]}(\partial \phi-\partial \chi)\right.} \\
& \left.+\psi_{\left[m_{1}\right.} \ldots \psi_{m_{4}} \partial^{2} X_{\left.m_{5}\right]}+i \psi_{m_{1}} \ldots \psi_{m_{5}}\left(\left(k^{\perp} \psi\right)(\partial \phi-\partial \chi)+\left(k^{\perp} \partial \psi\right)\right)\right) e^{i k^{\perp} X}(w) \\
& \equiv-\left[\oint \frac{d z}{2 i \pi} e^{\phi-\chi} G_{m a t t e r}(z), e^{\phi} \psi_{m_{1}} \ldots \psi_{m_{5}} e^{i k^{\perp} X}:(w)\right]
\end{aligned}
$$

where $G_{\text {matter }}(z)=\frac{1}{2} \psi_{m} \partial X^{m}(z)$ is the worldsheet matter supercurrent. So we have shown that adding $C_{2}(w)$ to $e^{\phi} \psi_{m_{1}} \ldots \psi_{m_{5}} e^{i k^{\perp} X}(w)$ indeed annihilates the first BRST noninvariance, originating from the commutator $\left[\oint \frac{d z}{2 i \pi} \gamma G_{m a t t e r}(z), e^{\phi} \psi_{m_{1}} \ldots \psi_{m_{5}} e^{i k^{\perp} X}(w)\right]$. Now let us find the expression for the $C_{1}(w)$ ghost counterterm which shall compensate for the non-invariance originating from the commutator of $e^{\phi} \psi_{m_{1}} \ldots \psi_{m_{5}} e^{i k^{\perp} X}(w)$ with the ghost supercurrent term of $Q_{B R S T}$ We will be looking for the ansatz for $C_{1}(w)$ in the form:

$$
C_{1}(w)=\left(c \hat{T}_{\chi}\right)_{7} \partial b b P_{\alpha \phi-\alpha \chi+\rho \sigma}^{(1)} e^{2 \phi-\chi} \psi_{m_{1}} \ldots \psi_{m_{5}}\left(\psi \partial^{2} X\right)^{\perp} e^{i k^{\perp} X}(w)
$$

with

$$
\left.T_{\chi}=\left((\partial \chi)^{2}+\lambda \partial^{2} \chi\right)\right)
$$

where $\alpha, \rho, \lambda$ are some real numbers which are to be determined from the condition that $C_{1}(w)$ has suitable commutation relations with $Q_{B R S T}$ to cancel the above mentioned BRST non-invariance; the weight 1 polynomial $P_{\alpha \phi-\alpha \chi+\rho \sigma}^{(1)}=\alpha \partial \phi-\alpha \partial \chi+\rho \partial \sigma$ is defined according to (24). Analogously to $(20)$, the operator $\left(c \hat{T}_{\chi}\right)_{7}$ is defined so that

$$
\left(c \hat{T}_{\chi}\right)_{n} A(w)=\lim _{u \rightarrow w}\left[\oint \frac{d z}{2 i \pi}(z-u)^{7} c T_{\chi}(z), A(w)\right]
$$

for any bosonic operator $\mathrm{A}$; if $\mathrm{A}$ is fermionic, the commutator is replaced by anticommutator, as usual. Let us start with calculating the commutator of $C_{1}(w)$ with the matter supercurrent term of $Q_{B R S T}$. It is important for our calculation that

$$
\begin{array}{r}
\gamma(z) P_{\alpha \phi-\alpha \chi+\rho \sigma}^{(1)}(w) \equiv e^{\phi-\chi}(z) P_{\alpha \phi-\alpha \chi+\rho \sigma}^{(1)}(w) \\
\sim \frac{\alpha-\alpha}{z-w}+O\left((z-w)^{0}\right) \sim O\left((z-w)^{0}\right)
\end{array}
$$


i.e. there is no singularity (simple pole) in the O.P.E. of $P_{\alpha \phi-\alpha \chi+\rho \sigma}^{(1)}$ with $\gamma$. Also using the fact that, as before, the $\left(c \hat{T}_{\chi}\right)_{7}$-operator commutes with the matter supercurrent term of $Q_{B R S T}$, we evaluate the commutator and obtain:

$$
\begin{aligned}
& {\left[\oint \frac{d z}{2 i \pi} e^{\phi-\chi} \psi_{m} \partial X^{m}(z), C_{1}(w)\right]} \\
& =\left(c \hat{T}_{\chi}\right)_{7} \partial b b P_{\alpha \phi-\alpha \chi+\rho \sigma}^{(1)} e^{3 \phi-2 \chi} \psi_{m_{1}} \ldots \psi_{m_{5}} e^{i k^{\perp} X}(w) \\
& \times\left[10 P_{\phi-\chi}^{(4)}+P_{\phi-\chi}^{(2)}\left[\left(i k \partial^{2} X\right)^{\perp}+(\partial \psi \psi)^{\perp}\right]\right. \\
& +P_{\phi-\chi}^{(1)}\left[\left(\partial X \partial^{2} X\right)^{\perp}+\left(\partial^{2} \psi \psi\right)^{\perp}\right] \\
& \left.+\frac{1}{2}\left(\partial^{2} \psi \partial \psi\right)^{\perp}+\frac{1}{3}\left(\partial^{3} \psi \psi\right)^{\perp}+i\left(k \partial^{2} X\right)^{\perp}(\partial \psi \psi)^{\perp}\right]
\end{aligned}
$$

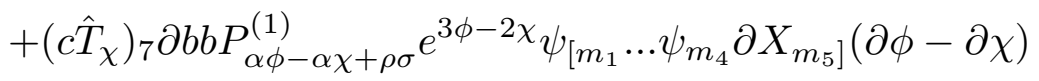

$$
\begin{aligned}
& +\psi_{\left[m_{1}\right.} \ldots \psi_{m_{4}} \partial^{2} X_{\left.m_{5}\right]} e^{i k^{\perp} X}(w) \equiv\left(c \hat{T}_{\chi}\right)_{7} L_{1}(w)
\end{aligned}
$$

Next, we need to evaluate the commutator $\left(c \hat{T}_{\chi}\right)_{7} L_{1}(w)$. Let us make several observations about (32) which simplify things significantly. The operator product of $c T_{\chi}(z)$ and $L_{1}(w)$ contains singular terms with maximum singularity order of $(z-w)^{-9}$ But because of the factor of $(z-u)^{7}$ in the definition $(37)$ of $\left(c \hat{T}_{\chi}\right)_{7}$ it is clear that the only non-vanishing contributions to this commutator will come from the the O.P.E. terms of $c T_{\chi}(z)$ and $L_{1}(w)$ having the singularity order of at least $(z-w)^{-8}$; any contributions originating from less singular terms of this O.P.E.will vanish after evaluating the contour integral, since they will contain factors of the type $\lim _{u \rightarrow w}(u-w)^{8-n}=0$ where $n$ is the order of the O.P.E. singularity. Therefore it is clear that out of all the terms that constitute the long expression (39) the only term contributing to the commutator $\left(c \hat{T}_{\chi}\right)_{7} L_{1}(w)$ is the one that contains the weight 4 ghost polynomial $P_{\phi-\chi}^{(4)}$ where, in accordance with the definition $(24)$

$$
\begin{array}{r}
P_{\phi-\chi}^{(4)}=\frac{1}{24}\left(\partial^{4} \phi-\partial^{4} \chi\right)+\frac{1}{6}\left(\partial^{3} \phi-\partial^{3} \chi\right)(\partial \phi-\partial \chi) \\
+\frac{1}{8}\left(\partial^{2} \phi-\partial^{2} \chi\right)^{2}+\frac{1}{4}\left(\partial^{2} \phi-\partial^{2} \chi\right)(\partial \phi-\partial \chi)^{2}+\frac{1}{24}(\partial \phi-\partial \chi)^{4}
\end{array}
$$

Fortunately for us there are no terms in (39) with the weight $3 P_{\phi-\chi}^{(3)}$ polynomial factor; if such terms were present, they would also have contributed to $\left(c \hat{T}_{\chi}\right)_{7} L_{1}(w)$, spoiling the entire construction. As for the terms with the $P_{\phi-\chi}^{(2)}$ factor, they all have maximum singularity order of $(z-w)^{-7}$ in the O.P.E. with $c T_{\chi}(z)$; terms with the $P_{\phi-\chi}^{(1)}$ give the maximum singularity order of $(z-w)^{-6}$, i.e. as has been explained above, these terms are irrelevant, as they do not contribute to the commutator. Having explained this all, let us 
finally evaluate the commutator of $\left(c \hat{T}_{\chi}\right)_{7}$ with $L_{1}(w)$, using $(37)$ and (38), as well as the O.P.E's:

$$
\begin{array}{r}
e^{\sigma}(z) P_{\alpha \phi-\alpha \chi+\rho \sigma}^{(1)}(w) \sim-\frac{\rho}{z-w} e^{\sigma}(w)+:\left(P_{\alpha \phi-\alpha \chi+\rho \sigma}^{(1)}-\rho \partial \sigma\right) e^{\sigma}:(w)+\ldots \\
=-\frac{\rho}{z-w} e^{\sigma}(w)+: P_{\alpha \phi-\alpha \chi}^{(1)} e^{\sigma}:(w)+\ldots \\
\partial \chi(z) \partial^{n} \chi(w) \sim \frac{n !}{(z-w)^{n+1}}+: \partial \chi \partial^{n} \chi:(w)+\ldots \\
\partial \chi(z) e^{2 \phi-\chi}(w) \sim-\frac{1}{z-w}+: \partial \chi e^{2 \phi-\chi}:(w)+\ldots
\end{array}
$$

etc.

We have:

$$
\begin{array}{r}
\left.\lim _{u \rightarrow w} 10 \oint \frac{d z}{2 i \pi}(z-u)^{7}: c\left((\partial \chi)^{2}+\lambda \partial^{2} \chi\right):(z) P_{\phi-\chi}^{(4)} \partial b b P_{\alpha \phi-\alpha \chi}^{(1)}\right) \\
\times e^{3 \phi-2 \chi} \psi_{m_{1} \ldots \psi_{m_{5}} e^{i k^{\perp} X}(w)} \\
=\lim _{u \rightarrow w} 10 \oint \frac{d z}{2 i \pi} \frac{(z-u)^{7}}{(z-w)^{8}} b e^{3 \phi-2 \chi} \psi_{m_{1} \ldots \psi_{m_{5}} e^{i k^{\perp} X}(w)} \\
\left\{3\left(P_{\alpha \phi-\alpha \chi+\rho \sigma}^{(1)}-\rho \partial \sigma\right)-\rho(2 \partial \phi-3 \partial \chi)+2 \alpha \partial \sigma-2 \alpha(\partial \phi-\partial \chi)\right\} \\
=10 P_{(\alpha-2 \rho) \phi+(3 \rho-\alpha) \chi+2 \alpha \sigma}^{(1)} b e^{3 \phi-2 \chi} \psi_{m_{1} \ldots \psi_{m_{5}} e^{i k^{\perp} X}(w)}
\end{array}
$$

Next,

$$
\begin{array}{r}
\lim _{u \rightarrow w} 10 \oint \frac{d z}{2 i \pi}(z-u)^{7}: c \partial^{2} \chi:(z) P_{\phi-\chi}^{(4)} \partial b b P_{\alpha \phi-\alpha \chi+\rho \sigma}^{(1)} \\
\times e^{3 \phi-2 \chi} \psi_{m_{1}} \ldots \psi_{m_{5}} e^{i k^{\perp} X}(w) \\
=10 P_{(5 \alpha-4 \rho) \phi-(4 \rho+5 \alpha) \chi}^{(1)} b e^{3 \phi-2 \chi} \psi_{m_{1}} \ldots \psi_{m_{5}} e^{i k^{\perp} X}(w)
\end{array}
$$

Adding these two contributions together gives:

$$
\begin{array}{r}
{\left[\oint \frac{d z}{2 i \pi} e^{\phi-\chi} \psi_{m} \partial X^{m}(z) ; C_{1}(w)\right] \equiv\left(c \hat{T}_{\chi}\right)_{7} L_{1}(w)} \\
=\lim _{u \rightarrow w} 10 \oint \frac{d z}{2 i \pi}(z-u)^{7}: c\left(\partial \chi \partial \chi+\lambda \partial^{2} \chi\right):(z) P_{\phi-\chi}^{(4)} \partial b b P_{\alpha \phi-\alpha \chi+\rho \sigma}^{(1)} \\
\times e^{3 \phi-2 \chi} \psi_{m_{1}} \ldots \psi_{m_{5}} e^{i k^{\perp} X}(w) \\
=10 P_{((\alpha-2 \rho)+\lambda(5 \alpha-4 \rho)) \phi+((3 \rho-\alpha)-\lambda(4 \rho+5 \alpha)) \chi+2 \alpha \sigma}^{(1)} b e^{3 \phi-2 \chi} \psi_{m_{1}} \ldots \psi_{m_{5}} e^{i k^{\perp} X}(w)
\end{array}
$$

Finally, we have to choose the coefficients $\alpha, \rho$ and $\lambda$ so that $\left(c \hat{T}_{\chi}\right)_{7} L_{1}(w)$ could annihilate the second BRST non-invariance (21) of the vertex $e^{\phi} \psi_{m_{1}} \ldots \psi_{m_{5}} e^{i k^{\perp} X}(w)$, originating from 
its commutation with the ghost supercurrent term of the $Q_{B R S T}$. The obvious condition for that is given by

$$
P_{((\alpha-2 \rho)+\lambda(5 \alpha-4 \rho)) \phi+((3 \rho-\alpha)-\lambda(4 \rho+5 \alpha)) \chi+2 \alpha \sigma}^{(1)}=P_{2 \phi-2 \chi-\sigma}^{(1)}(w)
$$

from which we immediately have

$$
\alpha=-\frac{1}{2}
$$

and the following system of two equations for $\rho$ and $\lambda$ :

$$
\begin{aligned}
& -\frac{1}{2}-2 \rho-\frac{5}{2} \lambda-4 \lambda \rho=2 \\
& \frac{1}{2}+3 \rho+\frac{5}{2} \lambda-4 \lambda \rho=-2
\end{aligned}
$$

which has the following solutions:

$$
\begin{array}{r}
\rho=0, \lambda=-1 \\
\rho=\frac{7}{8}, \lambda=-\frac{15}{8}
\end{array}
$$

These two solutions correspond to physically identical gauge choices for $C_{1}$. It is natural to choose the gauge first solution so that

$$
T_{\chi}=\partial \chi \partial \chi-\partial^{2} \chi
$$

is just twice the stress-energy tensor for the $\chi$-field; and the expression for $C_{1}$ is obtained to be:

$$
C_{1}(w)=\left(c \hat{T}_{\chi}\right)_{7} P_{-\frac{1}{2} \phi+\frac{1}{2} \chi}^{(1)} \partial b b e^{2 \phi-\chi} \psi_{m_{1}} \ldots \psi_{m_{5}}\left(\psi \partial^{2} X\right)^{\perp} e^{i k^{\perp} X}
$$

Because of the factor of 10 in (32) this operator should of course be multiplied by the appropriate numerical factor (given by $-\frac{1}{20}$ ) to compensate the BRST non-invariance. To summarize (15), (44) and (50), we have shown that

$$
-\frac{1}{20}\left[\oint \frac{d z}{2 i \pi} e^{\phi-\chi} \psi_{m} \partial X^{m}(z), C_{1}(w)\right]=-\frac{1}{4}\left[\oint \frac{d z}{2 i \pi} e^{2 \phi-2 \chi} b(z) ; e^{\phi} \psi_{m_{1}} \ldots \psi_{m_{5}} e^{i k^{\perp} X}(w)[\right.
$$

Finally, to prove that $-\frac{1}{20} C_{1}(w)$ is indeed the operator to insure (along with $C_{2}(w)$ ) the BRST-invariance of the picture +1 five-form, we still need to show that $C_{1}(w)$ commutes with all other terms in $Q_{B R S T}$. Let us start with the commutator $\left[\oint \frac{d z}{2 i \pi} e^{2 \phi-2 \chi} b(z), C_{1}(w)\right]$. This commutator vanishes since, as usual,

$$
\left\{\oint \frac{d z}{2 i \pi} e^{2 \phi-2 \chi} b(z),\left(c \hat{T}_{\chi}\right)_{7}\right\}=0
$$


and

$$
\begin{array}{r}
b(z): \partial b b:(w) \sim(z-w)^{2} e^{-3 \sigma}(w)+O\left((z-w)^{3}\right) \\
: e^{2 \phi-2 \chi}:(z): e^{2 \phi-2 \chi}:(w) \sim(z-w)^{-2} e^{4 \phi-3 \chi}+O\left((z-w)^{-1}\right) \\
\left.e^{2 \phi-2 \chi} P^{(} 1\right)_{-\frac{1}{2} \phi+\frac{1}{2} \chi}(w) \sim O\left((z-w)^{0}\right)
\end{array}
$$

Therefore

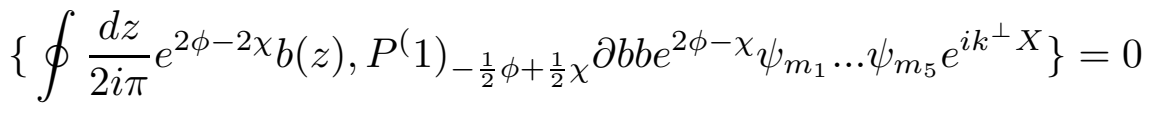

and hence

$$
\left[\oint \frac{d z}{2 i \pi} e^{2 \phi-2 \chi} b(z) ; C_{1}(w)\right]=0
$$

Next, let us focuse on the commutator of $C_{1}(w)$ with $\oint \frac{d z}{2 i \pi}\left(c T_{g h o s t}-b: c \partial c\right)$ of $Q_{B R S T}$. Again,

$$
\left\{\oint \frac{d z}{2 i \pi}\left(c T_{\text {ghost }}-b: c \partial c:\right)(z)\left(c \hat{T}_{\chi}\right)_{7}(w)\right\}=0
$$

since

$$
\left(c T_{\text {ghost }}-b: c \partial c:\right)(z)\left(c \hat{T}_{\chi}\right)_{7}(w) \sim: c \partial c:(w) \times \lim _{u \rightarrow w}(u-w)^{4}+O\left((u-w)^{5}\right)=0
$$

Next, it is easy to check that

$$
\begin{array}{r}
\left\{\oint \frac{d z}{2 i \pi}\left(c T_{\text {ghost }}-b: c \partial c\right)(z), P^{\left.(1)_{-\frac{1}{2} \phi+\frac{1}{2} \chi} \partial b b e^{2 \phi-\chi} \psi_{m_{1}} \ldots \psi_{m_{5}}\left(\psi \partial^{2} X\right)^{\perp} e^{i k^{\perp} X}(w)\right\}}\right. \\
=G_{\phi, \chi, \sigma}^{(4)} b e^{2 \phi-\chi} \psi_{m_{1}} \ldots \psi_{m_{5}}\left(\psi \partial^{2} X\right)^{\perp} e^{i k^{\perp} X}(w)
\end{array}
$$

where $G_{\phi, \chi, \sigma}^{(4)}$ is the conformal weight 4 polynomial consisting of derivatives of $\phi, \chi$ and $\sigma$, which exact form is not important and is not given here for the sake of brevity. It is easy to see from the simple dimensiaonal analysis that the operator product between $c T_{\chi}:(z)$ and $G_{\phi, \chi, \sigma}^{(4)}(w)$ must be given by

$$
c T_{\chi}(z) G_{\phi, \chi, \sigma}^{(4)}(w) \sim \frac{a}{(z-w)^{6}} c(w)+O\left((z-w)^{3}\right)
$$

where $a$ is some number, i.e. the most singular term in this O.P.E is of order of $(z-w)^{-6}$ Therefore

$$
\begin{array}{r}
\left(c \hat{T}_{\chi}\right)_{7} G_{\phi, \chi, \sigma}^{(4)} b e^{2 \phi-\chi} \psi_{m_{1}} \ldots \psi_{m_{5}}\left(\psi \partial^{2} X\right)^{\perp} e^{i k^{\perp} X}(w) \\
=\lim _{u \rightarrow w} \oint \frac{d z}{2 i \pi}(z-u)^{7} c T_{\chi}(z) G_{\phi, \chi, \sigma}^{(4)} b e^{2 \phi-\chi} \psi_{m_{1}} \ldots \psi_{m_{5}}\left(\psi \partial^{2} X\right)^{\perp} e^{i k^{\perp} X}(w) \\
=e^{2 \phi-\chi} \psi_{m_{1}} \ldots \psi_{m_{5}}\left(\psi \partial^{2} X\right)^{\perp} e^{i k^{\perp} X}(w) \times \lim _{u \rightarrow w} \oint \frac{d z}{2 i \pi}(z-u)^{7}(z-w)^{-7} \\
=7 e^{2 \phi-\chi} \psi_{m_{1}} \ldots \psi_{m_{5}}\left(\psi \partial^{2} X\right)^{\perp} e^{i k^{\perp} X}(w) \times \lim _{u \rightarrow w}(u-w)=0
\end{array}
$$


so we have just established

$$
\left[\oint \frac{d z}{2 i \pi}\left(c T_{\text {ghost }}-b: c \partial c:\right)(z) ; C_{1}(w)\right]=0
$$

- To conclude our derivation, we finally have to show that $C_{1}$ commutes with the last remaining piece of $Q_{B R S T}$ given by $\oint \frac{d z}{2 i \pi} c T_{\text {matter }}(z)$. Again, as previously,

$$
\left\{\oint \frac{d z}{2 i \pi} c T_{\text {matter }}(z)\left(c \hat{T}_{\chi}\right)_{7}(w)\right\}=0
$$

Next,

$$
\begin{aligned}
& \left\{\oint \frac{d z}{2 i \pi} c T_{\text {matter }}(z), P_{-\frac{1}{2} \phi+\frac{1}{2} \chi}^{(1)} \partial b b e^{2 \phi-\chi} \psi_{m_{1}} \ldots \psi_{m_{5}}\left(\psi \partial^{2} X\right)^{\perp} e^{i k^{\perp} X}(w)\right\} \\
& =b e^{2 \phi-\chi} \psi_{m_{1}} \ldots \psi_{m_{5}}\left(\psi \partial^{2} X\right)^{\perp} e^{i k^{\perp} X}(w) \\
& \times\left(2 i(k \psi)^{\perp} P_{\sigma}^{(5)}+2(\partial X \psi)^{\perp} P_{\sigma}^{(4)}+5\left(\partial^{2} X \psi\right)^{\perp} P_{\sigma}^{(3)}+\left(\partial^{3} X \psi\right)^{\perp} P_{\sigma}^{(2)}\right. \\
& +\frac{1}{3}\left(\partial^{4} X \psi\right)^{\perp} P_{\sigma}^{(1)}+\frac{1}{12}\left(\partial^{5} X \psi\right)^{\perp} \\
& +i\left(\partial^{2} X \psi\right)^{\perp}(k \partial X)^{\perp} P_{\sigma}^{(2)}+i\left(\partial^{2} X \psi\right)^{\perp}\left(k \partial^{2} X\right)^{\perp} P_{\sigma}^{(1)}+\frac{i}{2}\left(\partial^{2} X \psi\right)^{\perp}\left(k \partial^{3} X\right)^{\perp} \\
& +\left(\partial^{2} X \partial \psi\right)^{\perp} P_{\sigma}^{(2)}+\frac{3}{4}\left(\partial^{2} X \partial^{2} \psi\right)^{\perp} P_{\sigma}^{(1)} \\
& \left.+\frac{1}{3}\left(\partial^{2} X \partial^{3} \psi\right)^{\perp}+\left(\partial^{2} X \psi\right)^{\perp}(\partial \psi \psi)^{\perp} P_{\sigma}^{(1)}+\left(\partial^{2} X \psi\right)^{\perp} \partial(\partial \psi \psi)^{\perp}\right\} \\
& +P_{-\frac{1}{2} \phi+\frac{1}{2} \chi}^{(1)} \partial b b e^{2 \phi-\chi}\left(\psi \partial^{2} X\right)^{\perp} \\
& \times\left\{\frac{5}{2} \psi_{m_{1}} \ldots \psi_{m_{5}} P_{\sigma}^{(3)}+\partial\left(\psi_{m_{1}} \ldots \psi_{m_{5}}\right) P_{\sigma}^{(2)}+\frac{3}{4} \partial^{2} \psi_{\left[m_{1}\right.} \psi_{m_{2}} \ldots \psi_{\left.m_{5}\right]} P_{\sigma}^{(1)}\right. \\
& \left.+\frac{1}{3} \partial^{3} \psi_{\left[m_{1}\right.} \psi_{m_{2}} \ldots \psi_{\left.m_{5}\right]}\right\} \\
& \equiv L_{2}(w)
\end{aligned}
$$

Now we have to show that $\left(c \hat{T}_{\chi}\right)_{7} L_{2}(w)=0$. The crucial point to observe is that the O.P.E of the polynomials $P_{\sigma}^{(n)}(w)$ with the field $c(z)$ cannot contain terms more singular than $(z-w)^{-1}$ for any $n$, even though the polynomials have conformal weight equal to $\mathrm{n}$. Indeed, since $c(z) c(w) \sim(z-w): c \partial c(w)+O\left((z-w)^{2}\right)$, i.e. the lowest order term in this O.P.E. is of order of $(z-w)$, it is clear that the product $\partial_{z}^{n} c(z) c(w) \sim 0\left((z-w)^{0}\right)$, i.e. nonsingular. But by definition $\partial_{z}^{n} c(z) \equiv n ! P_{\sigma}^{(n)} c(z)$ and therefore there can be no singularities other that a simple pole in the O.P.E of $P_{\sigma}^{(n)}(w)$ and $c(z)$ Then, since the O.P.E of $T_{\chi}$ 
with $L_{2}(w)$ has the most singular part of order of $(z-w)^{-3}$ and $c(z) b(w) \sim(z-w)^{-1}$ we have

$$
\begin{gathered}
c T_{\chi}(z) L_{2}(w) \sim \frac{1}{(z-w)^{5}} \\
\left(c \hat{T}_{\chi}\right)_{7} L_{2}(w) \sim \lim _{u \rightarrow w} \oint \frac{d z}{2 i \pi}(z-u)^{7}\left((z-w)^{-5}+O\left((z-w)^{4}\right)+\ldots\right) \\
\sim \lim _{u \rightarrow w}\left((u-w)^{4}+O\left((u-w)^{5}\right)\right)=0
\end{gathered}
$$

and therefore

$$
\left\{\oint \frac{d z}{2 i \pi} c T_{\text {matter }}(z), C_{1}(w)\right\}=0
$$

Summing up (50),(51),(55),(66) we obtain

$$
-\frac{1}{20}\left[Q_{B R S T}, C_{1}(w)\right] \equiv=-\frac{1}{4}\left[\oint \frac{d z}{2 i \pi} e^{2 \phi-2 \chi} b(z), e^{\phi} \psi_{m_{1}} \ldots \psi_{m_{5}} e^{i k^{\perp} X}(w)\right.
$$

We have shown that the operator given by $-\frac{1}{20} C_{1}(w)$ indeed compensates for the second BRST non-invariance of $e^{\phi} \psi_{m_{1}} \ldots \psi_{m_{5}} e^{i k^{\perp} X}(w)$ and using (19),(34),(50) and (67) we see that the total combination $e^{\phi} \psi_{m_{1}} \ldots \psi_{m_{5}} e^{i k^{\perp} X}(w)-2 C_{2}(w)-\frac{1}{20} C_{1}(w)$ is BRST-invariant. To summarize our calculation we have shown that the BRST-invariant expression for the 5 -form operator at the +1 -picture is given by:

$$
\begin{aligned}
& V_{5}^{(+1)}(w)=\oint \frac{d w}{2 i \pi}\left\{e^{\phi} \psi_{m_{1}} \ldots \psi_{m_{5}} e^{i k^{\perp} X}(w)\right. \\
& -2 \hat{b_{3}} c \partial c e^{\chi} \partial \chi\left(\psi_{m_{1}} \ldots \psi_{m_{5}}(\psi \partial X)^{\perp}\right. \\
& +\psi_{\left[m_{1}\right.} \ldots \psi_{m_{4}} \partial X_{\left.m_{5}\right]}(\partial \phi-\partial \chi) \\
& \left.+\psi_{\left[m_{1}\right.} \ldots \psi_{m_{4}} \partial^{2} X_{\left.m_{5}\right]}+i \psi_{m_{1}} \ldots \psi_{m_{5}}\left(\left(k^{\perp} \psi\right)(\partial \phi-\partial \chi)+\left(k^{\perp} \partial \psi\right)\right)\right) e^{i k^{\perp} X}(w) \\
& \left.\left.-\frac{1}{20}\left(c \hat{T}_{\chi}\right)_{7} P^{(1}\right)_{-\frac{1}{2} \phi+\frac{1}{2} \chi} \partial b b e^{2 \phi-\chi} \psi_{m_{1}} \ldots \psi_{m_{5}}\left(\psi \partial^{2} X\right)^{\perp} e^{i k^{\perp} X}\right\}
\end{aligned}
$$

This concludes the derivation of the BRST-invariant 5-form vertex operator at the +1 picture as well as our discussion of the BRST-invariance of the brane-like states.

\section{BRST non-triviality of the Brane-like states}

The specific property of the picture -3 five-form vertex operator in the unintegrated form, as well as of the two-form (2) is that their picture changing transformation vanishes (for the two-form this is true only in he zero momentum case). This may cause one to suspect that these two states are actually BRST-trivial. Indeed, there exists a conventional wisdom, implying that if a BRST-invariant operator $V$ satisfies $: \Gamma V:=0$ then $V$ is BRSTtrivial, i.e. there exists an operator A such that $V=\left\{Q_{B R S T}, V\right\}$. Below we will show 
that this conventional wisdom is wrong and comment on what actually goes wrong in the standard proof of this claim. First of all, that this conventional wisdom is wrong is easy to see from the following simple example. Consider an open string three-point function $<V_{F}^{-3 / 2}(k) V_{F}^{-1 / 2}(-k) V_{B}(0)>$ where

$$
\begin{array}{r}
V_{F}^{-3 / 2}(k)=u_{\alpha}(k) c e^{-\frac{3}{2} \phi} \Sigma_{\alpha} e^{i k X}\left(z_{1}\right) \\
V_{F}^{-1 / 2}(-k)=v_{\alpha}(-k) c e^{-\frac{1}{2} \phi} \Sigma_{\alpha} e^{-i k X}\left(z_{1}\right) \\
V_{B}(0)=e_{m}(0) c \partial X^{m}
\end{array}
$$

Here $V_{F}^{-1 / 2}(-k)$ is the standard Ramond vertex at the $\frac{-1}{2}$-picture with the usual onshell condition $k \gamma_{\alpha \beta}^{m} u^{\beta}(k)=0$, where $\gamma^{m}$ are symmetric $16 \times 16$ ten-dimensional gammamatrices. $V_{B}(0)$ is a photon vertex operator which for simplicity we take at zero momentum (therefore there are no transversality conditions on the $e_{m}(0)$ polarization vector); and $V_{F}^{-3 / 2}(k)$ is the Ramond vertex operator at the picture $-3 / 2$ which polarization spinor is choosen to impose the condition $k \gamma_{\alpha \beta}^{m} u^{\beta}(k)=0$, even though in principle such a condition is not necessary in case of the picture $-3 / 2$ Ramond operator (unlike the picture $-1 / 2$ case). If, however, we do choose such a condition, it is easy to see that the picture-changing transformation of $V_{F}^{-3 / 2}(k)$ vanishes:

$$
: \Gamma V_{F}^{-3 / 2}(k):=i\left(k_{m} \gamma_{\alpha \beta}^{m} u_{\alpha}\right) c e^{-\frac{1}{2} \phi} \Sigma_{\beta} e^{i k X}=0
$$

due to the condition that we have imposed on the polarization spinor $u(k)$ However, it is elementary to see that the three-point correlation function, described above, does not vanish but is equal to:

$$
<V_{F}^{-3 / 2}(k) V_{F}^{-1 / 2}(-k) V_{B}(0)>=i(k e(0))(u(k) v(-k))
$$

But since all the operators: $V_{F}^{-3 / 2}(k), V_{F}^{-1 / 2}(-k)$ and $V_{B}(0)$ are BRST-invariant and their three-point function is nonzero, this means that the operator $V_{F}^{-3 / 2}(k)$ cannot be BRSTtrivial, even though its picture-changing transform vanishes. Having illustrated the clear error in the conventional wisdom on this simple example, let us discuss in more details the origin of this error. The standard proof of the claim, on which the mistaken wisdom is based, is the following. One takes a BRST-invariant operator $V$, such that

$$
: \Gamma V:=0
$$


and multiplies this identity by an inverse picture-changing operator

$$
\Gamma^{-1}=\frac{2}{3} c e^{\chi-2 \phi} \partial \chi
$$

. Then, by claiming

$$
: \Gamma^{-1} \Gamma V:=0
$$

and

$$
: \Gamma^{-1} \Gamma:=\left\{Q_{B R S T}, A\right\}
$$

where $\mathrm{A}$ is some operator, and using the BRST-invariance of $\mathrm{V}$, one writes

$$
0=: \Gamma^{-1} \Gamma V:=V+\left\{Q_{B R S T}, A\right\} V=V+\left\{Q_{B R S T}, A V\right\}
$$

and hence claiming the BRST triviality of any operator $\mathrm{V}$ with vanishing picture-changing transform:

$$
V=-\left\{Q_{B R S T}, A V\right\}
$$

It is easy to see, however, that such a proof, although standard, contains consistency problems and contradictions, which make it impossible to apply it to the case of the picture -3 five-form. First of all, it must be remembered that the identity (69) is true only in the normally ordered sense; strictly speaking, there are also higher order terms in the full O.P.E and in general one is not allowed to drop these terms in the product of three operators $\Gamma^{-1}, \Gamma$ and $V$, substituting the product $\Gamma^{-1} \Gamma V$ with its normally ordered part. Such a substitution, neglecting the higher order terms in the O.P.E of $\Gamma$ and $V$, is particularly incorrect if $\Gamma^{-1}$ has a singular O.P.E. with $\mathrm{V}$ - and this is exactly what happens in case of $\Gamma^{-1}$ and the picture -3 five-form (2).

More generally, substituting $A B$ with $: A B$ : in the product of three operators $\mathrm{A}, \mathrm{B}$ and $\mathrm{C}$ is not correct. Moreover, if these operators are such that : $B C:=0$, this would not necessarily imply $: A B C:=0$ To illustrate the above remarks, consider a simple example $A=e^{\phi} b, B=e^{-\phi} c, C=e^{n \phi}$ where $\mathrm{n}$ is some positive number. Clearly,

$$
: A B: \sim 1
$$

but at the same time

$$
: B C: \sim 0
$$


Now if one follows the the logic of the standard proof of (77), neglecting the higher order terms, one can multiply the identity (79) by A to obtain

$$
A B C \sim 0
$$

At the same time, using (78) one would get

$$
: A B C: \sim C
$$

and hence

$$
C \sim 0
$$

which clearly is a contradiction. As was noted above, the reason for this contradiction is that when one is computing the normally ordered expression for $\mathrm{ABC}$, one cannot substitute $\mathrm{AB}$ with its normally ordered part if the O.P.E. between $\mathrm{A}$ and $\mathrm{C}$ (or between $\mathrm{B}$ and $\mathrm{C}$ ) is singular. Since this is clearly fulfilled in case of the inverse picture-changing operator and the picture -3 five-form (2), the vanishing picture-changing transformation of this five-form does NOT imply its BRST triviality.

BRST non-triviality condition, however, does impose strong constraints on the propagation of the 5-form, constraining it to propagate in lower dimensional subspace of the $R^{10}$ Below we shall give a detailed analysis of these constraints. To start with, there are two and only two possible sources of potential BRST triviality threat for the picture -3 five-form (2). Namely, this threat is coming from commutations of dimension 1 primary fields $U_{1}$ and $U_{2}$, such that commutators of $U_{1}$ with matter supercurrent part of $Q_{B R S T}$ and those of $U_{2}$ with the ghost supercurrent part of the BRST charge. The only possible expression for $U_{1}$ is given by:

$$
U_{1}(w)=\oint \frac{d w}{2 i \pi} e^{\chi-4 \phi} \partial \chi \psi_{m_{1}} \ldots \psi_{m_{5}}(\psi \partial X) e^{i k^{\perp} X}(w)
$$

Indeed, as is easy to check

$$
\left[\oint \frac{d w}{2 i \pi} \gamma(\psi \partial X) ; U_{1}(w)\right]=e^{-3 \phi} \partial \chi \psi_{m_{1}} \ldots \psi_{m_{5}} e^{i k^{\perp} X}(w)
$$

and

$$
\left[\oint \frac{d w}{2 i \pi} \gamma^{2} b, U_{1}(w)\right]=0
$$

Therefore in order to insure the BRST non-triviality of the picture -3 five-form operator one must insure that $U_{1}$ does not commute with the stress-energy part of $Q_{B R S T}$. This in 
turn imposes constraints on the propagation of the five-form, restricting $k^{\perp}$ to propagate in five-dibensional subspace, orthogonal to the one spanned by the indices $m_{1} \ldots m_{5}$. Indeed, the supercurrent factor $(\psi \partial X)$ in the expression for the $U_{1}$-operator clearly directed orthogonally to the subspace spanned by $m_{1} \ldots m_{5}$, since $\psi_{m_{i}}^{2}=0$. Therefore it is easy to see that

$$
\left[\oint \frac{d w}{2 i \pi}\left(c\left(T_{\text {matter }}+T_{\text {ghost }}\right)-b: c \partial c\right)(z), U_{1}(w)=e^{\chi-4 \phi} \partial \chi \psi_{m_{1}} \ldots \psi_{m_{5}} e^{i k^{\perp} X}(w) \times\left(\psi^{t} k_{t}^{\perp}\right)\right.
$$

where $t$ denotes the directions orthogonal to $m_{1} \ldots m_{5}$. Therefore the last commutator is non-vanishing only if the scalar product $\left(\psi^{t} k_{t}^{\perp}\right)$ is non-zero, i.e. $k^{\perp}$ is also directed orthogonally to the $m_{1} \ldots m_{5}$ directions. Equivalently, one may say that if $k^{\perp}$ is orthogonal to $m_{1} \ldots m_{5}$, then the $U_{1}$-operator is not a primary field and therefore does not commute with $\oint \frac{d w}{2 i \pi}\left(c\left(T_{\text {matter }}+T_{\text {ghost }}\right)-b: c \partial c\right)(z)$ of $Q_{B R S T}$. We conclude that BRST non-triviality condition constrains the propagation of the five-form vertex operator to the five-dimensional subspace of $R^{10}$. Therefore one may think of the five-form as of a multidimensional analogue of the discrete states that are well-known to occur in two-dimensional quantum gravity. Finally, the second threat of the BRST triviality comes from the operator

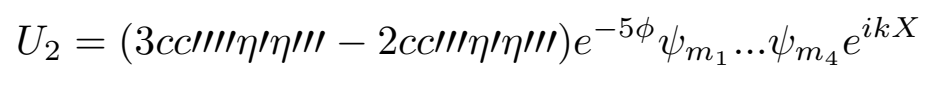

(where we denoted $\eta=e^{\chi}$ ) since, as one can straightforwardly check this operator is a primary field commuting with $\oint \frac{d w}{2 i \pi}\left(c\left(T_{\text {matter }}+T_{\text {ghost }}\right)-b: c \partial c\right)(z)$ of $Q_{B R S T}$. It also commutes with the matter supercurrent part $\oint \frac{d w}{2 i \pi} \gamma(\psi \partial X)$ of $Q_{B R S T}$, while a simple analysis of conformal dimensions and of the ghost numbers easily shows that the ghost supercurrent part of $Q_{B R S T}$, given by $\oint \frac{d w}{2 i \pi} \gamma^{2} b$ has a simple pole in the O.P.E. with the integrand of the $U_{2}$-operator and, up to some numerical coefficient, produces exactly the five-form vertex operator, which would imply its BRST-triviality. However, the crucial point is that this numerical coefficient turns out to be equal to zero, and therefore, as there are clearly no other triviality threats, the five-form is NOT BRST exact and IS physical. Let us give a detailed proof that indeed this numerical coefficient is zero, which means that $Q_{B R S T}$ simply commutes with $U_{2}$ and therefore the $U_{2}$ poses no triviality threat to the five-form. The proof follows below. Using the standard bosonization formulae:

$$
\begin{gathered}
c=e^{\sigma} \\
b=e^{-\sigma} \\
\eta=e^{\chi} \\
\gamma=e^{\phi-\chi}
\end{gathered}
$$


Then, as it is straightforward to check, the normal ordered bosonization formulae for the two terms constituting the $U_{2}$-operator (without the $e^{-5 \phi}$-factor and the matter part) are given by:

$$
\begin{array}{r}
W_{1}=3 c c \prime \prime \prime \prime \eta \prime \eta \prime \prime=3\left\{\left(-\frac{1}{2}\left(e^{2 \sigma}\right) \prime \prime \prime-3\left(\sigma \prime \prime e^{2 \sigma}\right) \prime\right)\left(\chi^{\prime \prime}-\chi^{\prime} \chi \prime\right) e^{2 \chi}\right\} \\
W_{2}=2 c c \prime \prime \prime \eta \prime \eta \prime \prime \prime=2\left\{\left(-\frac{3}{4}\left(e^{2 \sigma}\right) \prime \prime-\frac{3}{2}(\sigma) \prime \prime e^{2 \sigma}\right)\left(-\frac{1}{4}\left(e^{2 \chi}\right) \prime \prime \prime+\frac{3}{2}\left(\chi^{\prime \prime} e^{2 \chi}\right) \prime\right)\right\}
\end{array}
$$

where $U_{2} \equiv e^{-5 \phi}\left(W_{1}-W_{2}\right) \psi_{m_{1}} \ldots \psi_{m_{5}} e^{i k X}$.

Now we need to evaluate the most singular terms (of order of $(z-w)^{-11}$ ) of the O.P.E of $W_{1}$ and $W_{2}$ with $\gamma^{2} b$ of $Q_{b r s t}$ because these terms are the only ones contributing to the simple pole $\left(\operatorname{as} e^{2 \phi}(z) e^{-5 \phi}(w) \sim(z-w)^{10} e^{-3 \phi}(w)\right)$ Using the O.P.E's

$$
\begin{array}{r}
\sigma \prime(z) e^{\alpha \sigma}(w) \sim \frac{\alpha}{z-w} e^{\alpha \sigma}(w)+\ldots \\
\sigma \prime \prime(z) e^{\alpha \sigma}(w) \sim-\frac{\alpha}{(z-w)^{2}} e^{\alpha \sigma}(w)+\ldots \\
e^{\alpha \sigma}(z) e^{\beta \sigma}(w) \sim(z-w)^{\alpha \beta} e^{(\alpha+\beta) \sigma}(w) \text { etc }
\end{array}
$$

and similarly for the exponents of the type $e^{\alpha \chi}$ we obtain:

$$
\begin{aligned}
e^{-2 \chi}(z)\left(\chi^{\prime \prime}-\chi^{\prime} \chi^{\prime}\right) e^{2 \chi}(w) \sim(z-w)^{-4}\left(\frac{2}{(z-w)^{2}}\right. & \left.-\frac{4}{(z-w)^{2}}\right)+\ldots \\
& =-2(z-w)^{-6}+\ldots \\
e^{-\sigma}(z):\left(-\frac{1}{2}\left(e^{2 \sigma}\right) \prime \prime \prime-3\left(\sigma \prime \prime e^{2 \sigma}\right) \prime\right)(w): \sim e^{\sigma}(w)\left\{-\frac{1}{2} \partial_{w}^{3}\left(\frac{1}{(z-w)^{2}}\right)\right. & -3 \partial_{w}\left(\frac{1}{(z-w)^{4}}\right) \\
& =-24(z-w)^{-5}
\end{aligned}
$$

and therefore

$: \gamma^{2} b:(z) W_{1}(w) \sim 3 \times(-2) \times(-24)(z-w)^{-5-6} c e^{2 \phi}(w)+\ldots=144(z-w)^{-11} c e^{2 \phi}(w)+\ldots$

Next, consider the O.P.E. of $\gamma^{2} b$ with $W_{2}$. Again, proceeding as above we have:

$$
\begin{aligned}
& e^{-\sigma}(z):\left(-\frac{3}{4}\left(e^{2 \sigma}\right) \prime \prime-\frac{3}{2}(\sigma) / / e^{2 \sigma}\right):(w) \\
& =-\frac{3}{4} \partial_{w}^{2}\left(\frac{1}{(z-w)^{2}}\right)-\frac{3}{2} \frac{1}{(z-w)^{4}}+\ldots=-\frac{6}{(z-w)^{4}}+\ldots \\
& e^{-2 \chi}(z):\left(-\frac{1}{4}\left(e^{2 \chi}\right) \prime \prime \prime+\frac{3}{2}\left(\chi^{\prime \prime} e^{2 \chi}\right) \prime\right):(w) \\
& =-\frac{1}{4} \partial_{w}^{3}\left(\frac{1}{(z-w)^{4}}\right)+\frac{3}{2} \partial_{w}\left(\frac{2}{(z-w)^{6}}\right)+\ldots=-\frac{12}{(z-w)^{7}}+\ldots
\end{aligned}
$$


and therefore

$$
\begin{array}{r}
: \gamma^{2} b:(z) W_{2}(w) \sim \frac{2 \times-6 \times-12}{(z-w)^{4+7}} c e^{2 \phi}(w)+\ldots \\
\quad=144(z-w)^{-11} c e^{2 \phi}(w)
\end{array}
$$

- Then, using the above commutation relations and the fact that $e^{2 \phi}(z) e^{-5 \phi}(w) \sim$ $(z-w)^{10} e^{-3 \phi}+\ldots$ we find that the total commutator of the $\mathrm{W}$-operator with the ghost supercurrent term of $Q_{b r s t}$ is given by:

$$
\begin{aligned}
& \oint d z \gamma^{2} b(z) W(w)=\oint e^{2 \phi-2 \chi-\sigma}(z) e^{-5 \phi}\left(W_{1}-W_{2}\right) \psi_{m_{1}} \ldots \psi_{m_{5}} e^{i k X}(w) \\
& =\oint \frac{d z}{z-w}\left(144 c e^{-3 \phi} \psi_{m_{1}} \ldots \psi_{m_{5}} e^{i k X}-144 c e^{-3 \phi} \psi_{m_{1}} \ldots \psi_{m_{5}} e^{i k X}\right)=0
\end{aligned}
$$

Therefore the total commutator of $Q_{B R S T}$ with $U_{2}$ is equal to zero and the five-form is proven to be BRST non-trivial (given its "transverse" five-dimensional propagation). Equivalently, the BRST non-triviality of the 5 -form at picture -3 can be proven by considering the tree-point function of $V_{5}^{(-3)}(k)$ with $V_{5}^{(+1)}(p)$ and $V_{B}(q)$ where $V_{B}$ is again a photonic vertex and $\mathrm{k}, \mathrm{p}$ and $\mathrm{q}$ are the corresponding momenta. For certainty, let us consider all the operators in the integrated form. Since all these operators are proven to be BRST invariant, the non-zero answer for the correlator will be a sufficient proof for the BRST non-triviality. Since expressions for both photonic operator in unintegrated form and $V_{5}^{(-3)}(k)$ contain no fermionic ghosts, only the first piece of $V_{5}^{(+1)}(k)$ without b and c fields will contribute to the three-point correlator. A simple computation gives:

$$
\begin{aligned}
& <V_{5}^{(-3)}(k) V_{5}^{(+1)}(p) V_{B}(q)> \\
& =<e^{-3 \phi} \psi_{m_{1}} \ldots \psi_{m_{5}} e^{i k X}\left(z_{1}\right) e^{\phi} \psi_{m_{1}} \ldots \psi_{m_{5}} e^{i p X}\left(z_{2}\right) e_{m}(q)\left(\partial X^{m}+i(q \psi) \psi^{m}\right) e^{i q X}\left(z_{3}\right)> \\
& =i\left(k^{\perp} e(q)\right)^{\perp} \times \frac{1}{\left(z_{1}-z_{2}\right)\left(z_{1}-z_{3}\right)\left(z_{2}-z_{3}\right)}
\end{aligned}
$$

The coordinate dependence cancels out after being multiplied by Koba-Nielsen's measure, so the final answer for the correlator is given by just $i\left(k^{\perp} e(q)\right)^{\perp} \neq 0$ which proves the BRST non-triviality of the five-form if $k=k^{\perp}$, i.e. is transverse to the $m_{1} \ldots m_{5}$ directions which define the polarization of the five-form - in complete agreement with our previous analysis. Technically, the transverse momentum dependense in the answer arises because for the longitudinal ones the contribution from the NSR fermionic part of $V_{B}(q)$ proportional to $(q \psi) \psi^{m}$ cancels the one from the bosonic part and therefore the total answer is zero; while for the transversely oriented momenta fermionic part of $V_{B}(q)$ does not contribute 
while the bosonic part gives non-zero contribution leading to the answer given above. This concludes the analysis of the BRST properties of the five-form vertex operators.

\section{Closed String Brane-like States and Effective Actions}

In this paper we have proven that the brane-like vertex operators define new physical states in superstring theory and are closely related to the non-perturbative brane dynamics. Closed string brane-like states can be constructed in a straightforward way taking the vertices (1) as holomorphic and antiholomorphic parts of closed string operators. Then various polarizations and the way one contracts indices in these operators would correspond to introducing various branes and brane configurations. As a simplest example consider a closed-string operator:

$$
V_{5}=\int d^{2} z \epsilon_{a_{1} \ldots a_{6}} \lambda\left(k^{\perp}\right) e^{-3 \phi-\bar{\phi}} \psi_{a_{1} \ldots} \psi_{a_{5}} \bar{\psi}_{a_{6}} e^{i k^{\perp} X}(z, \bar{z})
$$

where the $10 \mathrm{~d}$ space-time index $m$ is split in the $4+6$ way: $m=(t, a) ; t=0, \ldots, 3, a=4, \ldots, 9$ and in this case $i k^{\perp} X \equiv k_{t} X^{t}$. As is easy to see, this closed string operator consists of a holomorphic picture -3 five-form and antiholomorphic photonic vertex at picture -1 (the latter may of course be taken at any picture). In this case, as is easy to see by direct generalization of our previous analysis, BRST invariance + non-triviality condition require that the momentum $k^{\perp}$ propagates in $t$-directions only, i.e. it is 4 -dimansional; $\lambda\left(k^{\perp}\right)$ is a space-time scalar field. The operator (97) has a $S O(1,3) \times S O(6)$ isometry, i.e. the one of a D3-brane background. Therefore it is natural to expect that inserting such an operator is equivalent to introducing D3-branes in the theory and simulating an effective geometry created by $D 3$ - branes. Any choice of the scalar function $\lambda\left(k^{\perp}\right)$ corresponds to particular distribution of the D3-branes in this picture. In particular, it turns out [2] that imposing certain constraints on the form of this scalar function, namely, choosing $\lambda\left(k^{\perp}\right) \sim \frac{1}{\left(k^{\perp}\right)^{4}}$ creates a near-horizon geometry of D3-branes playing a crucial role in AdS/CFT correspondence [5], [6], [7] and can be used for constructing the NSR superstring theory on $A d S_{5} \times S^{5}$ [8], [2] To conclude this paper we would like to present the result of computing the low-energy effective action corresponding to sigma-model with the closed-string $V_{5}$-field, i.e. the effective action for the space-time $\lambda$-field It turns out that this action has a DBI structure and the answer is given by:

$$
S_{e f f}(\lambda)=\int d^{4} X e^{-\varphi} \sqrt{\operatorname{det}\left(\eta_{s t}+\partial_{s} \lambda \partial_{t} \lambda\right)}
$$

where the integration is taken over 4-dimensional subspace defined by the $X^{s}$ coordinates $(s, t=0, \ldots 3) \eta$ is Minkowski tensor and $\varphi$ is the dilaton field. It is remarkable that the 
closed string sigma-model with the operator (97) has a low-energy action with open-string dilaton dependence (as it should be for D-branes). Such an anomalous dilaton dependence in the effective action (98) must be deeply related to the hidden RR-origin of the brane-like states, observed in this paper. The explanation for such an unusual dilaton coupling, as well as detailed derivation of the effective action (98) will be given in our next paper which is currently in progress.

\section{Acknowledgements}

This work has been supported by the Academy of Finland under the Project No. 163394. 


\section{References}

[1] D.Polyakov, hep-th/0005094, Class.Quant.Grav. bf 18:1979, 2001

[2] D.Polyakov, hep-th/9812044,Phys.Lett. bf B470:103-111

[3] I.R. Klebanov, A.M. Polyakov, Mod.Phys.Lett.A6:3273-3281,1991

[4] D.Friedan, E.Martinec, S.Shenker, Nucl.Phys.B271:93,1986

[5] J.Maldacena, Adv.Theor.Math.Phys.2 (1998) 231-252, hep-th/9711200

[6] S.Gubser,I.Klebanov, A.M.Polyakov, Phys.Lett. B428:105-114

[7] E.WittenAdv.Theor.Math.Phys.2:253-291,1998

[8] R.Kallosh, A.Tseytlin,J.High Energy Phys.9810:016,1998 